\title{
Alcohol, Age, and Mortality: Estimating Selection Bias Due to Premature Death
}

\author{
TIMOTHY S. NAIMI, M.D., M.P.H., ${ }^{a, b, c, *}$ LYNDSEY A. STADTMUELLER, B.S., ${ }^{b}$ TANYA CHIKRITZHS, PH.D., ${ }^{d}$ \\ TIM STOCKWELL, PH.D., ${ }^{c}$ JINHUI ZHAO, PH.D., ${ }^{c}$ ANNIE BRITTON, PH.D., ${ }^{e}$ RICHARD SAITZ, M.D., M.P.H., ${ }^{a, b} \&$ \\ ADAM SHERK, M.A. ${ }^{c}$ \\ ${ }^{a}$ Clinical Addiction Research and Education Unit, Section of General Internal Medicine, Boston Medical Center, Boston, Massachusetts \\ ${ }^{b}$ Department of Community Health Sciences, Boston University School of Public Health, Boston, Massachusetts \\ ${ }^{c}$ Canadian Institute for Substance Use Research, Victoria, British Columbia, Canada \\ ${ }^{d}$ National Drug Research Institute, Curtin University, Perth, Australia \\ eUniversity College of London, London, England
}

\begin{abstract}
Objective: Alcohol use causes approximately 10\% of deaths among adults ages $20-65$ in the United States. Although previous research has demonstrated differential age-related risk relationships, it is difficult to estimate the magnitude of selection bias attributable to premature mortality based on existing cohort studies, the average age of which is greater than 50 years. The objective of our study was to assess the distribution of mortality-related harms and benefits from alcohol among adults ages 20 and older in comparison with the distribution among those older than age 50. Method: Data from the Centers for Disease Control and Prevention's Alcohol-Related Disease Impact software application from 2006-10 were used to determine the distribution of alcohol-attributable deaths (AADs) and the years of potential life lost (YPLLs) that was caused or prevented by alcohol for 54 conditions by
\end{abstract}

15-year age groupings $(20-34,35-49,50-64,65+)$ in the United States. We also determined the proportion of net deaths and YPLLs occurring in each age group, overall and by cause of death. Results: Adults ages 20-49 years experienced $35.8 \%$ of the deaths and $58.4 \%$ of the YPLLs caused by alcohol, whereas the same group accrued only $4.5 \%$ of AADs and $14.2 \%$ of YPLLs gained. Overall, $46.3 \%$ of the total net deaths and $64.7 \%$ of the net YPLLs occurred among those ages 20-49; adding net deaths occurring among those ages 20-49 to those occurring after age 50 results in an $86.3 \%$ relative increase in net deaths. Conclusions: Because of premature mortality, alcohol-mortality associations based on cohort studies may underestimate negative health consequences compared with those among the general population. (J. Stud. Alcohol Drugs, 80, 000-000, 2019)
$\mathrm{T}$ HE RELATIONSHIP BETWEEN alcohol consumption and mortality is increasingly controversial, but it has important implications for public health and for the development of alcohol policy strategies. Data informing alcohol-mortality relationships is derived principally from observational studies (e.g., cohort studies). Most cohort studies used to study the health effects of alcohol consumption were designed to study risk factors for chronic disease outcomes such as cardiovascular disease and cancer and typically enroll adults of middle age and older. Because alcohol may be related to premature mortality (i.e., relative to the typical age at cohort enrollment), this is a potentially important source of selection bias (Naimi et al., 2017).

Because alcohol consumption has health effects across the life course, it would be ideal to measure mortality from studies that are representative of ages during which alcohol is consumed. However, because alcohol consumption is initi-

Received: September 11, 2018. Revision: November 16, 2018.

No author has any conflict of interest or relevant commercial interests to disclose. Funding was from general salary support from the institutions listed above.

*Correspondence may be sent to Timothy Naimi at the Clinical Addiction Research and Education Unit, Boston Medical Center, 801 Massachusetts Ave., Room 2046, Boston, MA 02118, or via email at: tim.naimi@bmc.org. ated by age 21 in more than $90 \%$ of drinkers in developed countries (Degenhardt et al., 2008), the initiation of alcohol consumption may occur decades before typical ages of enrollment among typical cohorts used to assess the health effects of alcohol. Deaths at relatively younger ages are rarely from chronic diseases (e.g., cardiovascular disease) for which alcohol may play a preventive role. Therefore, assuming that relatively young persons are more likely to die from alcohol consumption than they are to die from a lack of drinking, enrolling relatively older persons in studies would be expected to minimize risk relationships (or maximize potential benefits) compared with an analysis that included all drinking initiates (Gmel et al., 2003; Naimi et al., 2017).

Previous studies have demonstrated that age influences the relationships between alcohol consumption and health outcomes, both in the aggregate and on a condition-specific basis (Britton \& McPherson, 2001; Global Burden of Disease 2016 Collaborators, 2018; Mäkelä, 1998; Marmet et al., 2014; Rehm et al., 2009; Shield \& Rehm, 2015; World Health Organization, 2014). Specifically, studies generally find that, in the aggregate (e.g., all-cause mortality), alcohol-related risk is higher among relatively younger persons compared with older persons. In addition, even on a condition-specific basis (e.g., coronary heart disease [CHD]), younger ages appear to be associated with greater risk (Zhao 
et al., 2017). This may be attributable to effect modification on the basis of age, age-related differences in patterning of consumption (most studies assess only average consumption levels), or selection bias from premature mortality as discussed above.

However, findings about alcohol-mortality relationships are rarely contextualized with respect to the age of study participants, and in fact information about the mean age or mean weighted age (in the case of meta-analyses) of study participants is rarely reported. This makes it difficult to assess the potential magnitude of selection bias from premature mortality. However, in recent meta-analyses conducted by members of our research team, one on all-cause mortality (Stockwell et al., 2016) and another on CHD (Zhao et al., 2017), the mean weighted age at cohort enrollment was in the early $50 \mathrm{~s}$ in both studies. This age should be similar to the typical age of subjects in other meta-analyses, since ours generally included the same studies as prior meta-analyses, plus several more recent studies. This offered the opportunity to estimate the potential magnitude of selection bias from alcohol-attributable premature mortality and years of life lost before and after age 50 using population-based health outcome data from the U.S. Centers for Disease Control and Prevention (CDC).

\section{Method}

Alcohol-attributable deaths (AADs) and years of potential life lost (YPLLs) data from all levels of alcohol use by age group and gender were obtained from the CDC's AlcoholRelated Disease Impact (ARDI) software application. A full description of the methods and references involved has been published on the ARDI website (CDC, 2015). In brief, the ARDI application estimates AADs and YPLLs for 54 medical conditions in the United States using cause of death data from the National Vital Statistics System and the Fatality Analysis Reporting System. The most recent version of ARDI covers deaths in the United States from 2006 to 2010, and was used for this analysis. Some conditions in ARDI are $100 \%$ alcohol attributable (e.g., alcoholic liver cirrhosis). Others rely on estimates of alcohol involvement from metaanalyses or complete samples (e.g., alcohol-related motor vehicle crashes). Most chronic conditions rely on populationattributable fraction calculations based on adjusted prevalence estimates of various levels of alcohol consumption and relative risk estimates from published meta-analyses.

ARDI provides default data tables of the harms (AADs and YPLLs caused by alcohol) and benefits (AADs and YPLLs prevented) for various levels of alcohol use gender and age categories $(0-19,20-34,35-49,50-64$, and 65+). We excluded deaths among the 0 - to 19 -year-old category since a high proportion of those deaths are caused by others' alcohol use (e.g., young passengers killed in alcohol-related crashes), and because most drinkers have begun consum- ing by age 20 (Degenhardt et al., 2008). To calculate the proportion of alcohol-attributable deaths from each disease occurring in any given age, we divided the number of deaths in that age group by the total number of deaths across all age groups and multiplied by 100 .

To also investigate the distribution of AADs and YPLLs for different causes of death, we divided the 54 conditions into categories of related conditions, as shown in Table 1. We combined the data given by ARDI for the harms and benefits to calculate the net proportion of AADs and YPPLs overall and for each of the disease categories.

\section{Results}

In the United States from 2006 to $2010,35.8 \%$ of AADs (i.e., deaths caused by alcohol) accrued to individuals 20-49 years of age, whereas only $4.5 \%$ of AADs prevented by alcohol accrued to this age group (Figure 1). Conversely, the $65+$ age group accounted for approximately $35 \%$ of AADs caused by alcohol versus approximately $80 \%$ of AADs prevented by alcohol. Men were disproportionately likely to experience AADs caused by alcohol at young ages compared with women, who were disproportionately likely to have AADs prevented by alcohol at older ages (e.g., 65+).

In terms of YPLLs, $58.4 \%$ of YPLLs caused by alcohol accrued to those ages $20-49$, whereas only $14.2 \%$ of YPLLs prevented (i.e., years of life saved) accrued to this age group (Figure 2). Those age 65 and older accounted for less than $15 \%$ of YPLLs caused by alcohol, but they accounted for more than 50\% of YPLLs prevented by alcohol. Similar sexspecific differences were found for YPLLs as for AADs.

AADs and YPLLs caused and prevented were combined to calculate the net AADs and net YPLLs for deaths from all conditions and for nine disease categories, and the percentage of AADs and YPPLs in each category was apportioned by age group (Table 1). Across all disease categories, 46.3\% $(35,883 / 77,391)$ of the net deaths and $64.7 \%(1,508,371 /$ $2,331,330)$ of the net YPLLs accrued to those ages 20-49. In terms of disease categories with a disproportionately large impact among relatively young ages, among deaths from transport, violence, and "other" disease categories, more than $50 \%$ of the net AADs and YPLLs accrued to those ages $20-49$ years. By contrast, approximately $90 \%$ of the net AADs and YPLLs caused by alcohol for cancer, and $100 \%$ of the net AADs and YPLLs prevented by alcohol for cardiovascular diseases, accrued to those age 50 and older.

\section{Discussion}

Although prior research has documented differential risk of alcohol-attributable harms on the basis of age (Global Burden of Disease 2016 Collaborators, 2018), this analysis examined the age distribution of deaths and years of potential life lost caused and prevented by alcohol in the United 
TABLE 1. Percentage of net ${ }^{a}$ deaths and life years lost attributable to alcohol, by age group and disease category, Alcohol Related Disease Impact application, 2006-2010

\begin{tabular}{|c|c|c|c|c|c|}
\hline Variable & $20-34$ & $35-49$ & $50-64$ & $65+$ & Total \\
\hline \multicolumn{6}{|c|}{ Net alcohol-attributable deaths by age group (years) } \\
\hline All causes & $19.00 \%$ & $27.30 \%$ & $33.70 \%$ & $20.00 \%$ & 77,391 \\
\hline Transport ${ }^{b}$ & $43.90 \%$ & $31.10 \%$ & $18.50 \%$ & $6.50 \%$ & 11,856 \\
\hline Violence $^{c}$ & $37.30 \%$ & $30.00 \%$ & $21.10 \%$ & $11.50 \%$ & 14,595 \\
\hline Other $^{d}$ & $27.20 \%$ & $40.60 \%$ & $25.40 \%$ & $6.80 \%$ & 8,677 \\
\hline Neurological ${ }^{e}$ & $6.50 \%$ & $19.10 \%$ & $32.20 \%$ & $42.30 \%$ & 989 \\
\hline Alcohol toxicity $f^{\prime}$ & $6.50 \%$ & $31.90 \%$ & $45.80 \%$ & $15.80 \%$ & 7,401 \\
\hline Injuries $^{g}$ & $5.80 \%$ & $8.90 \%$ & $14.20 \%$ & $71.20 \%$ & 9,656 \\
\hline Gastrointestinal $^{h}$ & $1.90 \%$ & $22.60 \%$ & $46.00 \%$ & $29.50 \%$ & 23,701 \\
\hline Cardiovascular ${ }^{i}$ & $0.50 \%$ & $-0.30 \%$ & $-14.20 \%$ & $-86.00 \%$ & $-13,109$ \\
\hline Cancer $^{j}$ & $0.40 \%$ & $6.30 \%$ & $32.90 \%$ & $60.40 \%$ & 13,625 \\
\hline \multicolumn{6}{|c|}{ Net years of potential life lost by age group (years) } \\
\hline All causes & $32.00 \%$ & $32.70 \%$ & $27.40 \%$ & $7.90 \%$ & $2,331,330$ \\
\hline Transport & $57.50 \%$ & $29.00 \%$ & $11.70 \%$ & $1.80 \%$ & 464,757 \\
\hline Violence & $52.00 \%$ & $30.10 \%$ & $14.30 \%$ & $3.50 \%$ & 532,483 \\
\hline Other & $38.10 \%$ & $41.40 \%$ & $18.40 \%$ & $2.10 \%$ & 311,794 \\
\hline Neurological & $14.80 \%$ & $30.50 \%$ & $34.30 \%$ & $20.40 \%$ & 21,985 \\
\hline Alcohol & $11.50 \%$ & $40.70 \%$ & $40.40 \%$ & $7.40 \%$ & 204,304 \\
\hline Injuries & $18.90 \%$ & $20.20 \%$ & $21.70 \%$ & $39.20 \%$ & 150,625 \\
\hline Gastrointestinal & $3.90 \%$ & $33.60 \%$ & $47.40 \%$ & $15.00 \%$ & 558,264 \\
\hline Cardiovascular & $2.40 \%$ & $-0.40 \%$ & $-30.20 \%$ & $-71.90 \%$ & $-135,615$ \\
\hline Cancer & $1.20 \%$ & $13.20 \%$ & $46.60 \%$ & $38.90 \%$ & 222,731 \\
\hline
\end{tabular}

${ }^{a}$ Negative numbers indicate net benefits - that is, deaths prevented or prevented years of life lost. ${ }^{b}$ Transport causes included air-space transport, motor vehicle non-traffic crashes, motor vehicle traffic crashes, other road vehicle crashes, and water transport. ${ }^{c}$ Violence causes included child maltreatment, homicide, and suicide. " "Other" causes included alcoholic myopathy, fetal alcohol syndrome, fetus and newborn affected by maternal use of alcohol, low birth weight/prematurity/intrauterine growth retardation, psoriasis, and spontaneous abortion. ${ }^{e}$ Neurological causes included alcoholic polyneuropathy, alcoholic psychosis, degeneration of nervous system due to alcohol, and epilepsy. ${ }^{f}$ Alcohol toxicity causes included alcohol abuse, alcohol dependence, alcohol poisoning, excessive blood alcohol level, and suicide by exposure to alcohol. ${ }^{g}$ Injury causes included drowning, fall injuries, fire injuries, firearm injuries, and occupational and machine injuries. ${ }^{h}$ Gastrointestinal causes included acute pancreatitis, alcohol-induced chronic pancreatitis, alcoholic gastritis, alcoholic liver disease, cholelithiasis, chronic hepatitis, chronic pancreatitis, esophageal varices, gastroesophageal hemorrhage, liver cirrhosis unspecified, and portal hypertension. ${ }^{i}$ Cardiovascular causes included alcohol cardiomyopathy, hypertension, ischemic heart disease, hemorrhagic stroke, ischemic stroke, and supraventricular cardiac dysrhythmia. ${ }^{j}$ Cancer causes included breast cancer (women only), esophageal cancer, laryngeal cancer, liver cancer, oropharyngeal cancer, and prostate cancer (men only).

States, with a particular focus on mortality occurring among people age 20 and older compared with that among persons age 50 and older. We focused on this comparison because the average age in most cohort studies used to examine alcoholmortality relationships is over age 50 (Di Castelnuovo et al., 2006; Stockwell et al., 2016; Zhao et al., 2017). Our findings show that those over age 50 experience more of the potential mortality benefits from alcohol consumption and fewer of the harms from alcohol consumption compared with those accrued to all those over age 20 (i.e., ages after which most persons have begun to drink alcohol). Therefore, assuming that study cohorts have a reasonably similar age distribution of deaths as comparably aged persons in the general population, our findings suggest that existing meta-analyses of the alcohol-mortality relationship underestimate alcohol-related risks compared with those for drinkers of all ages. For individual conditions rather than all-cause mortality, the age distribution of their alcohol-attributable death would affect the degree of premature mortality.

Premature mortality is a type of selection bias, because deceased persons cannot be enrolled in cohort studies. The impact of this bias is related to the proportion of alcohol's mortality effect that occurs before the age of cohort enroll- ment (Naimi et al., 2017). In this study, more than one third of deaths caused by alcohol occurred among individuals younger than age 50 , whereas the vast majority of deaths prevented by alcohol accrued to those age 50 and older (e.g., $>90 \%$ of deaths and YPLLs prevented by cardiovascular disease are captured among those 50 and older). In terms of net deaths from all causes (total deaths caused minus those prevented), almost half (46.3\%) occurred before age 50, whereas $53.7 \%$ occurred afterward. Therefore, adding deaths that occur from ages 20 to 49 to those that occur after age 50 represents an $86.3 \%$ relative increase in the net number of alcohol-attributable deaths (i.e., the relative increase between 53.7 and 100.0). Compared with AADs, YPLLs caused by alcohol were skewed even younger, and YPLLs prevented by alcohol skewed even older. For example, adding the net YPLLs among those ages 20-49 to those that occur at age 50 or older results in a $250 \%$ relative increase in the net YPLLs.

It is tempting to say that this issue could be addressed by carefully contextualizing findings from cohort studies on the basis of age. However, findings from studies of established drinkers might not be the same as effects among older persons who might begin to drink, or purposefully continue to 


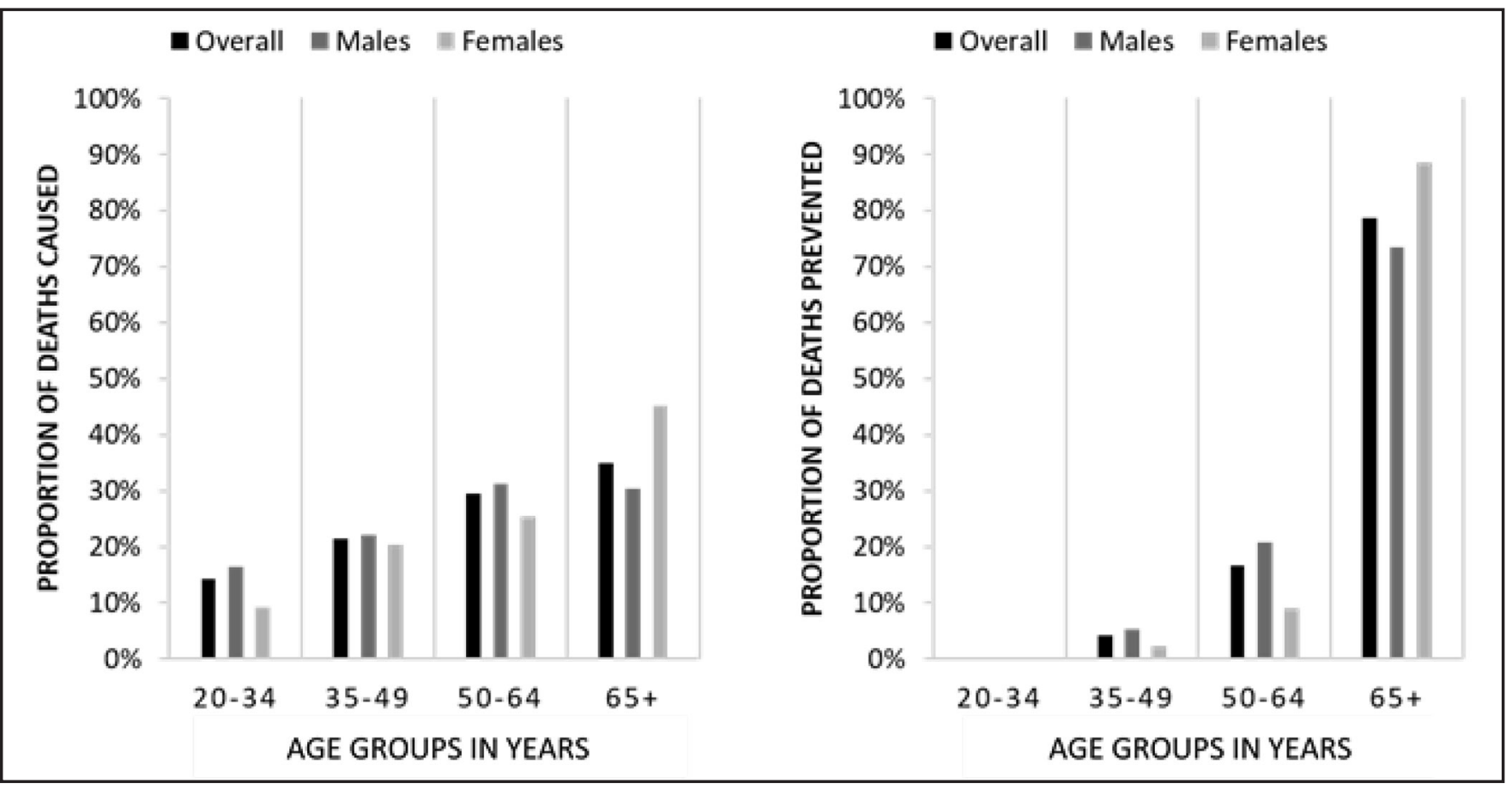

FIGURE 1. Proportion of alcohol-attributable deaths caused (left) and prevented (right) by age group in years and sex, United States, $2006-2010$.

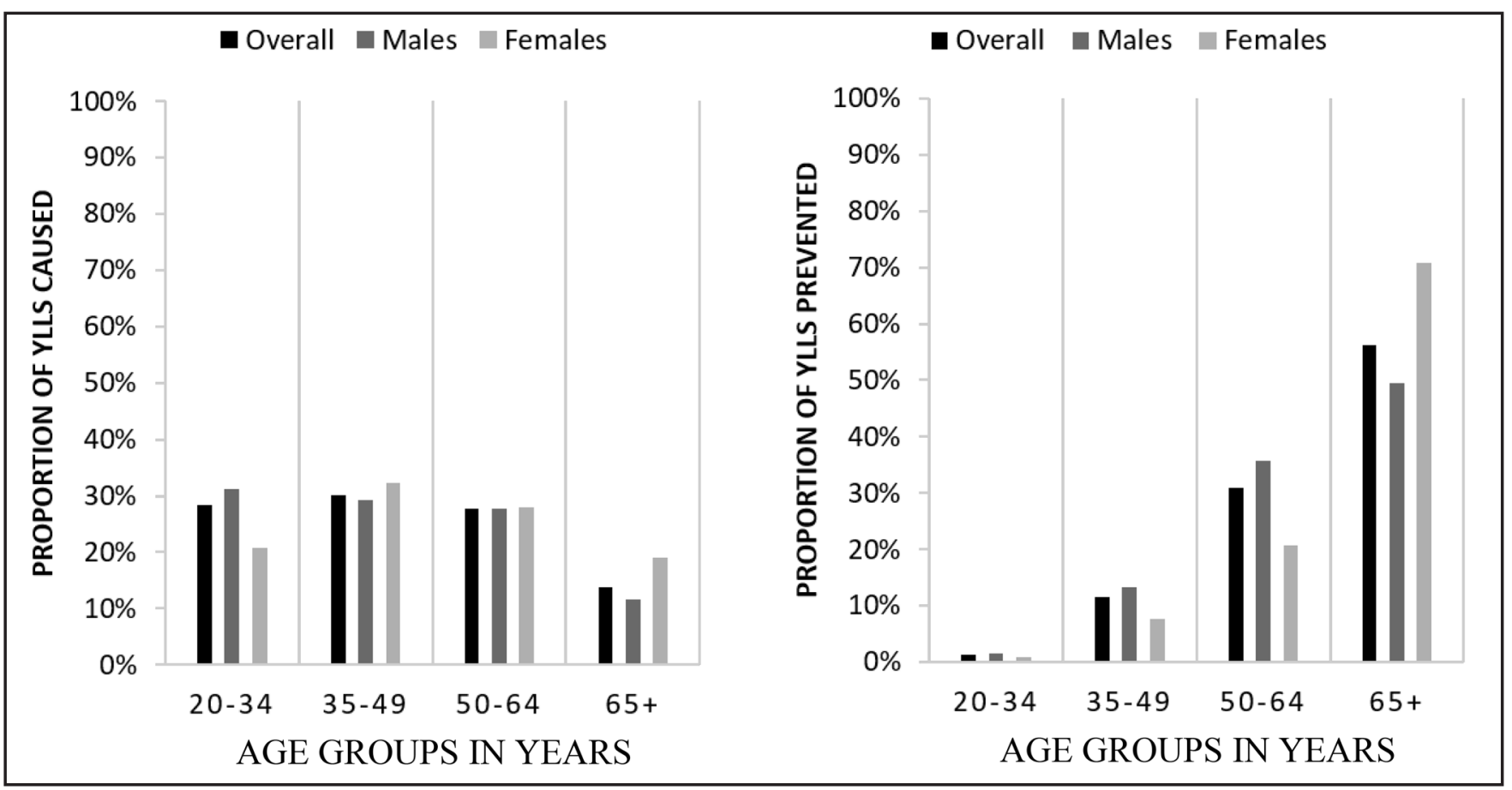

FIGURE 2. Proportion of alcohol-attributable years of potential life lost (YLLS) caused (left) and prevented (right) by age group in years and sex, United States, 2006-2010. 
drink, in order to prolong life. One reason for this is that those who are established drinkers at age 50 are "survivors" of their alcohol consumption who might have been healthier or have had safer drinking patterns compared with all persons who began to drink (Gmel et al., 2003; Haber et al., 2016; Naimi et al., 2017; Staff \& Maggs, 2017). Conversely, nondrinkers who start to drink in middle age would include some who are more vulnerable to alcohol and who might have already died had they begun drinking earlier in life.

A recent meta-analysis of the relationship of alcohol consumption with CHD mortality found different relationships on the basis of age. The meta-analysis included 45 studies, approximately 3 million participants, and 65,000 deaths (Zhao et al., 2017). Among cohorts with a mean age at enrollment of 56 years or older, there was significant protection from CHD mortality for low- (adjusted relative risk $[\mathrm{RR}]=0.81,95 \%$ confidence interval $[\mathrm{CI}][0.69,0.95])$ and medium-volume consumption $(\mathrm{RR}=0.77,95 \%$ CI $[0.66$, 0.90]). However, there was no significant protection for cohorts whose mean age at enrollment was 55 years or less $(\mathrm{RR}=0.95,95 \%$ CI $[0.75,1.21]$ for low-volume drinkers, $\mathrm{RR}=1.04,95 \% \mathrm{CI}[0.81,1.34]$ for medium-volume drinkers). Of note, cohorts in the studies selected for the latter analysis were all followed up to a mean age of at least 60 years and many considerably older - that is, ages at which coronary heart disease is more likely to occur.

This study is subject to caveats and limitations. This was a study in the United States, and so it is not necessarily generalizable to other developed nations. However, U.S. studies contribute more data than any other country to meta-analyses of total mortality and cardiovascular disease, and our findings of relatively large proportions of deaths occurring before age 50, and of beneficial effects occurring after age 50 , are consistent with global estimates and those from other developed nations (Britton \& McPherson, 2001; Mäkelä, 1998; Marmet et al., 2014; Rehm et al., 2009; Shield \& Rehm, 2015; World Health Organization, 2014).

For some disease categories, alcohol-attributable fraction estimates rely on relative risk estimates from meta-analyses and so are likely subject to the same issues of selection bias discussed in this article, as well as other limitations such as confounding. However, for conditions that are classified by ARDI as fully alcohol attributable (e.g., alcohol-related motor vehicle crash fatalities, other unintentional injuries), ARDI uses direct estimates that do not require relative risks, and these conditions also tend to affect people at ages that would precede eligibility for most cohort studies. The ARDI system does not apportion deaths or YPLLs solely on the basis of average consumption levels, so it is not possible to determine the relative differences in possible age-related selection bias among those with various patterns of consumption (i.e., "moderate" vs. heavy drinkers, binge drinkers, etc.; Bagnardi et al., 2008). Last, since the development of the ARDI application, there are an increased number of condi- tions that have been identified as being affected by alcohol. It is unclear how including other conditions would have affected the results.

It is important to note that selection bias arising from premature mortality is just one type of selection bias that may occur in observational studies. Former drinker bias - that is, including former drinkers in the nondrinking group along with lifetime abstainers - has been previously recognized as an important type of bias (Chikritzhs et al., 2009; Fillmore et al., 2007). With older age of study participants comes increased opportunity for drinkers to become former drinkers and introduces the potential for a type of selection bias that is often referred to as "sick quitter" bias, a bias that has received considerable attention in the scientific literature (Shaper et al., 1988; Wannamethee \& Shaper, 1998). In addition, alcohol-related morbidity (e.g., use disorder, other associated mental health conditions, alcohol-related injuries with residual sequelae) may mean that affected individuals are less likely to be able to participate, or be eligible to participate, as members of study cohorts (Jousilahti et al., 2005).

Based on recent evidence, the notion of protective effects from moderate alcohol consumption, particularly for all-cause mortality, is increasingly controversial (Global Burden of Disease 2016 Collaborators, 2018; Holmes et al., 2014; Stockwell et al., 2016; Wood et al., 2018). Overall, this study adds to the literature questioning protective effects for alcohol on all-cause mortality. However, it is important to acknowledge that there are many reasons why people choose to drink or not to drink alcohol apart from its health effects, and that most who choose to drink can do so with relatively low risk.

\section{Acknowledgments}

The authors acknowledge Marissa Esser, M.P.H., from the Centers for Disease Control and Prevention for her assistance with technical aspects of the Alcohol Related Disease Impact application.

\section{References}

Bagnardi, V., Zatonski, W., Scotti, L., La Vecchia, C., \& Corrao, G. (2008). Does drinking pattern modify the effect of alcohol on the risk of coronary heart disease? Evidence from a meta-analysis. Journal of Epidemiology and Community Health, 62, 615-619. doi:10.1136/ jech.2007.065607

Britton, A., \& McPherson, K. (2001). Mortality in England and Wales attributable to current alcohol consumption. Journal of Epidemiology and Community Health, 55, 383-388. doi:10.1136/jech.55.6.383

Centers for Disease Control and Prevention. (2015). Alcohol-Related Disease Impact (ARDI). Retrieved from https://nccd.cdc.gov/DPH_ARDI/ default/default.aspx

Chikritzhs, T., Fillmore, K., \& Stockwell, T. (2009). A healthy dose of scepticism: Four good reasons to think again about protective effects of alcohol on coronary heart disease. Drug and Alcohol Review, 28, 441-444. doi:10.1111/j.1465-3362.2009.00052.x

Degenhardt, L., Chiu, W.-T., Sampson, N., Kessler, R. C., Anthony, J. C., Angermeyer, M., . . . Wells, J. E. (2008). Toward a global view of 
alcohol, tobacco, cannabis, and cocaine use: Findings from the WHO World Mental Health Surveys. PLoS Medicine, 5, e141. doi:10.1371/ journal.pmed.0050141

Di Castelnuovo, A., Costanzo, S., Bagnardi, V., Donati, M. B., Iacoviello, L., \& de Gaetano, G. (2006). Alcohol dosing and total mortality in men and women: An updated meta-analysis of 34 prospective studies. Archives of Internal Medicine, 166, 2437-2445. doi:10.1001/archinte.166.22.2437

Fillmore, K. M., Stockwell, T., Chikritzhs, T., Bostrom, A., \& Kerr, W. (2007). Moderate alcohol use and reduced mortality risk: Systematic error in prospective studies and new hypotheses. Annals of Epidemiology, 17, Supplement 5, S16-S23. doi:10.1016/j.annepidem.2007.01.005

Global Burden of Disease 2016 Collaborators (2018). Alcohol use and burden for 195 countries and territories, 1990-2016: A systematic analysis for the Global Burden of Disease Study 2016. The Lancet, 392, 1015-1035. doi:10.1016/S0140-6736(18)31310-2

Gmel, G., Gutjahr, E., \& Rehm, J. (2003). How stable is the risk curve between alcohol and all-cause mortality and what factors influence the shape? A precision-weighted hierarchical meta-analysis. European Journal of Epidemiology, 18, 631-642. doi:10.1023/A:1024805021504

Haber, J. R., Harris-Olenak, B., Burroughs, T., \& Jacob, T. (2016). Residual effects: Young adult diagnostic drinking predicts late-life health outcomes. Journal of Studies on Alcohol and Drugs, 77, 859-867. doi:10.15288/jsad.2016.77.859

Holmes, M. V., Dale, C. E., Zuccolo, L., Silverwood, R. J., Guo, Y., Ye, Z., . . Casas, J. P., \& the InterAct Consortium. (2014). Association between alcohol and cardiovascular disease: Mendelian randomisation analysis based on individual participant data. BMJ, 349, g4164. doi:10.1136/ bmj.g4164

Jousilahti, P., Salomaa, V., Kuulasmaa, K., Niemelä, M., \& Vartiainen, E. (2005). Total and cause specific mortality among participants and non-participants of population based health surveys: A comprehensive follow up of 54372 Finnish men and women. Journal of Epidemiology and Community Health, 59, 310-315. doi:10.1136/jech.2004.024349

Mäkelä, P. (1998). Alcohol-related mortality by age and sex and its impact on life expectancy: Estimates based on the Finnish death register. European Journal of Public Health, 8, 43-51. doi:10.1093/eurpub/8.1.43

Marmet, S., Rehm, J., Gmel, G., Frick, H., \& Gmel, G. (2014). Alcoholattributable mortality in Switzerland in 2011-Age-specific causes of death and impact of heavy versus non-heavy drinking. Swiss Medical Weekly, 144, w13947. doi:10.4414/smw.2014.13947
Naimi, T. S., Stockwell, T., Zhao, J., Xuan, Z., Dangardt, F., Saitz, R., . . Chikritzhs, T. (2017). Selection biases in observational studies affect associations between 'moderate' alcohol consumption and mortality. Addiction, 112, 207-214. doi:10.1111/add.13451

Rehm, J., Mathers, C., Popova, S., Thavorncharoensap, M., Teerawattananon, Y., \& Patra, J. (2009). Global burden of disease and injury and economic cost attributable to alcohol use and alcohol-use disorders. The Lancet, 373, 2223-2233. doi:10.1016/S0140-6736(09)60746-7

Shaper, A. G., Wannamethee, G., \& Walker, M. (1988). Alcohol and mortality in British men: Explaining the U-shaped curve. The Lancet, 332, 1267-1273. doi:10.1016/S0140-6736(88)92890-5

Shield, K. D., \& Rehm, J. (2015). Global risk factor rankings: The importance of age-based health loss inequities caused by alcohol and other risk factors. BMC Research Notes, 8, 231. doi:10.1186/ s13104-015-1207-8

Staff, J., \& Maggs, J. (2017). Alcohol and cigarette use from ages 23 to 55: Links with health and well-being in the long-term National Child Development Study. Journal of Studies on Alcohol and Drugs, 78, 394-403. doi:10.15288/jsad.2017.78.394

Stockwell, T., Zhao, J., Panwar, S., Roemer, A., Naimi, T., \& Chikritzhs, T. (2016). Do "moderate" drinkers have reduced mortality risk? A systematic review and meta-analysis of alcohol consumption and allcause mortality. Journal of Studies on Alcohol and Drugs, 77, 185-198. doi:10.15288/jsad.2016.77.185

Wannamethee, S. G., \& Shaper, A. G. (1998). Alcohol, coronary heart disease and stroke: An examination of the J-shaped curve. Neuroepidemiology, 17, 288-295. doi:10.1159/000026182

Wood, A. M., Kaptoge, S., Butterworth, A. S., Willeit, P., Warnakula, S., Bolton, T., . . . Danesh, J., \& the Emerging Risk Factors Collaboration/ EPIC-CVD/UK Biobank Alcohol Study Group. (2018). Risk thresholds for alcohol consumption: Combined analysis of individual-participant data for 599912 current drinkers in 83 prospective studies. The Lancet, 391, 1513-1523. doi:10.1016/S0140-6736(18)30134-X

World Health Organization. (2014). Global status report on alcohol and health, 2014. Geneva, Switzerland: Author. Retrieved from https://www. who.int/substance_abuse/publications/alcohol_2014/en/

Zhao, J., Stockwell, T., Roemer, A., Naimi, T., \& Chikritzhs, T. (2017). Alcohol consumption and mortality from coronary heart disease: An updated meta-analysis of cohort studies. Journal of Studies on Alcohol and Drugs, 78, 375-386. doi:10.15288/jsad.2017.78.375 